\title{
Total Synthesis of Coprinol
}

Muthiah Suresh $^{\mathrm{a}}$, Navin Kumar ${ }^{\mathrm{a}}$, Gorre Veeraraghavaiah ${ }^{\mathrm{b}}$, Sunit Hazra ${ }^{\mathrm{a}}$, and Raj Bahadur Singh ${ }^{\mathrm{a}}$

${ }^{a}$ Centre for Applied Chemistry, Central University of Jharkhand, Brambe, Ranchi-835205, India

${ }^{b}$ School of Chemistry, University of Hyderabad, Central university P.O., Hyderabad-500046, India 


\section{SUPPORTING INFORMATION}

\section{Contents}

Page

3 ' ${ }^{1} \mathrm{H}$ NMR (500MHz, $\mathrm{CDCl}_{3}$ ) Spectrum of compound (4)

$4{ }^{13} \mathrm{C}$ NMR (125 MHz, $\mathrm{CDCl}_{3}$ ) Spectrum of compound (4)

$5{ }^{1} \mathrm{H}$ NMR (400MHz, $\mathrm{CDCl}_{3}$ ) Spectrum of compound (6)

$6{ }^{13} \mathrm{C}$ NMR (125MHz, $\mathrm{CDCl}_{3}$ ) Spectrum of compound (6)

$7{ }^{1} \mathrm{H}$ NMR (500MHz, $\mathrm{CDCl}_{3}$ ) Spectrum of compound (3)

$8{ }^{13} \mathrm{C}$ NMR (100MHz, $\mathrm{CDCl}_{3}$ ) Spectrum of compound (3)

$9{ }^{1} \mathrm{H}$ NMR (500MHz, $\mathrm{CDCl}_{3}$ ) Spectrum of compound (7)

$10{ }^{13} \mathrm{C}$ NMR (125MHz, $\mathrm{CDCl}_{3}$ ) Spectrum of compound (7)

$11{ }^{1} \mathrm{H}$ NMR (500MHz, $\mathrm{CDCl}_{3}$ ) Spectrum of compound (2)

$12{ }^{13} \mathrm{C}$ NMR (125 MHz, $\mathrm{CDCl}_{3}$ ) Spectrum of compound (2)

$13{ }^{1} \mathrm{H}$ NMR (500MHz, $\mathrm{CDCl}_{3}$ ) Spectrum of compound (8)

$14{ }^{13} \mathrm{C}$ NMR (125 MHz, $\mathrm{CDCl}_{3}$ ) Spectrum of compound (8)

$15{ }^{1} \mathrm{H}$ NMR (500MHz, $\mathrm{CDCl}_{3}$ ) Spectrum of compound (11)

$16{ }^{13} \mathrm{C}$ NMR (125 MHz, $\mathrm{CDCl}_{3}$ ) Spectrum of compound (11)

$17{ }^{1} \mathrm{H}$ NMR (500MHz, $\mathrm{CD}_{3} \mathrm{OD}$ ) Spectrum of compound (1)

$18{ }^{13} \mathrm{C}$ NMR (125 MHz, CD ${ }_{3} \mathrm{OD}$ ) Spectrum of compound (1)

$19{ }^{1} \mathrm{H}$ NMR (500MHz, $\mathrm{CDCl}_{3}$ ) Spectrum of compound (9) 
$20{ }^{13} \mathrm{C}$ NMR (125 MHz, $\mathrm{CDCl}_{3}$ ) Spectrum of compound (9)

$21{ }^{1} \mathrm{H}$ NMR (500MHz, $\mathrm{CDCl}_{3}$ ) Spectrum of compound (10)

$22{ }^{13} \mathrm{C}$ NMR (125 MHz, $\mathrm{CDCl}_{3}$ ) Spectrum of compound (10) 


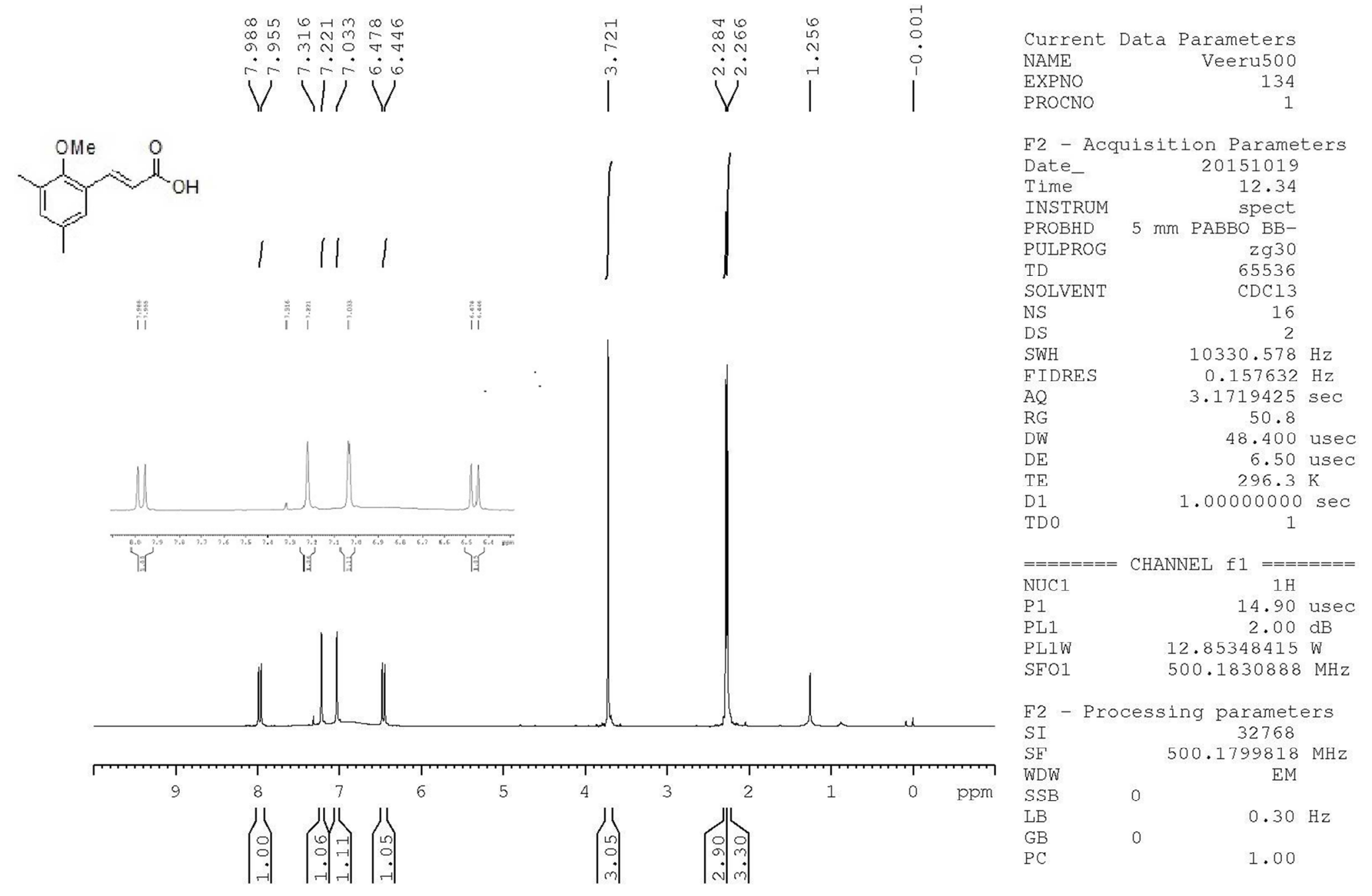

${ }^{1} \mathrm{H}$ NMR (500MHz, $\mathrm{CDCl}_{3}$ ) Spectrum of compound (4) 


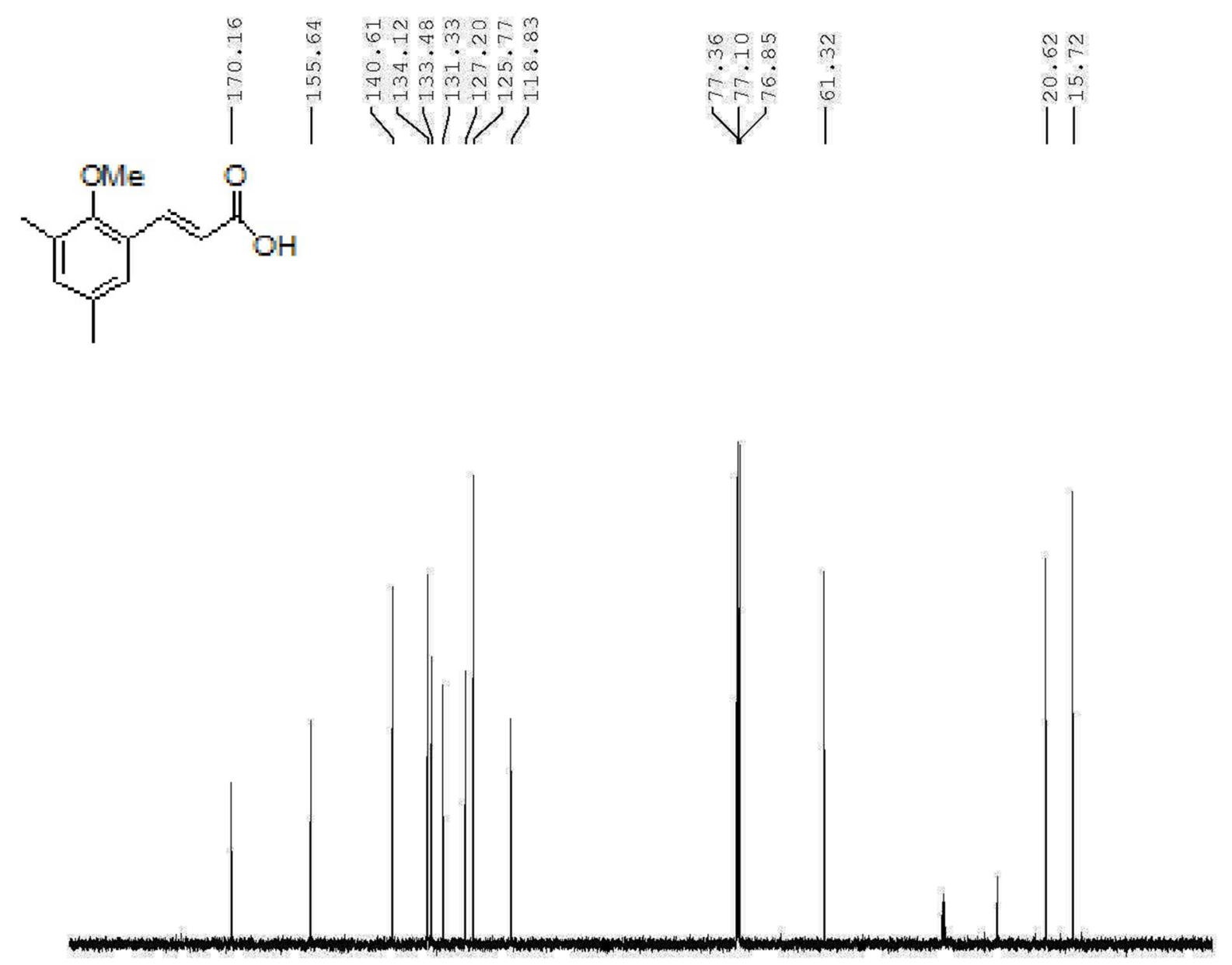

Current Data Parameters

EXPNO

F2 - Acquisition Paraneter

F2 - Acquisition Paramete
Date_20151019

Time

INSTRUM spect.

PULPROG

TD

SOLVEN
NS
DS

DS

FIDRES
AQ

$\mathrm{AQ}$
$\mathrm{RG}$

$R G$
$D W$
$D E$
$T E$

TE

D11
TDO

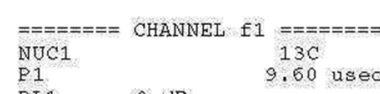
$\begin{array}{ll}\text { PL1W } & 75.36135101 \mathrm{~W} \\ \text { SLO1 } & 125.7829381 \mathrm{MHz}\end{array}$

$== \pm== \pm==$ CHANNEI f2 $========$ $== \pm== \pm=$ CHANNEL
CPDPRG 12 waltz16

$\begin{array}{lc}\text { NUC2 } & 1 \mathrm{H} \\ \text { PCPD2 } & 80.00 \mathrm{usec} \\ \text { PI2 } & 2.00 \mathrm{~dB}\end{array}$ $\begin{array}{lr}\text { PL2 } & 2.00 \mathrm{~dB} \\ \text { PL12 } & 16.50 \mathrm{~dB}\end{array}$

12.85348415 W

$\begin{array}{ll}\text { PL12W } & 0.45605880 \mathrm{~W} \\ \text { FL13W } & 0.45605880 \mathrm{~W}\end{array}$ $\begin{array}{ll}\text { FL13W } & 0.45605880 \mathrm{~W} \\ \mathrm{SFO2} & 500.1820002 \mathrm{MHz}\end{array}$

F2 - Processing parameters

$\begin{array}{lr}\mathrm{SI} & 32768 \\ \mathrm{SF} & 125.7703715 \mathrm{MHz}\end{array}$ WDW

LB $\quad 1.00 \mathrm{~Hz}$

$\mathrm{GB}$
$\mathrm{PC}$

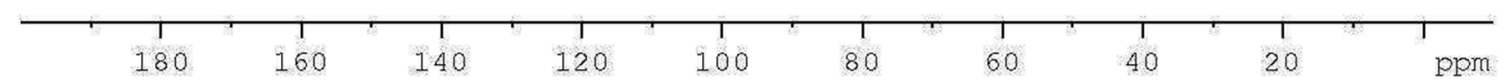

${ }^{13} \mathrm{C}$ NMR (125 MHz, $\mathrm{CDCl}_{3}$ ) Spectrum of compound (4) 


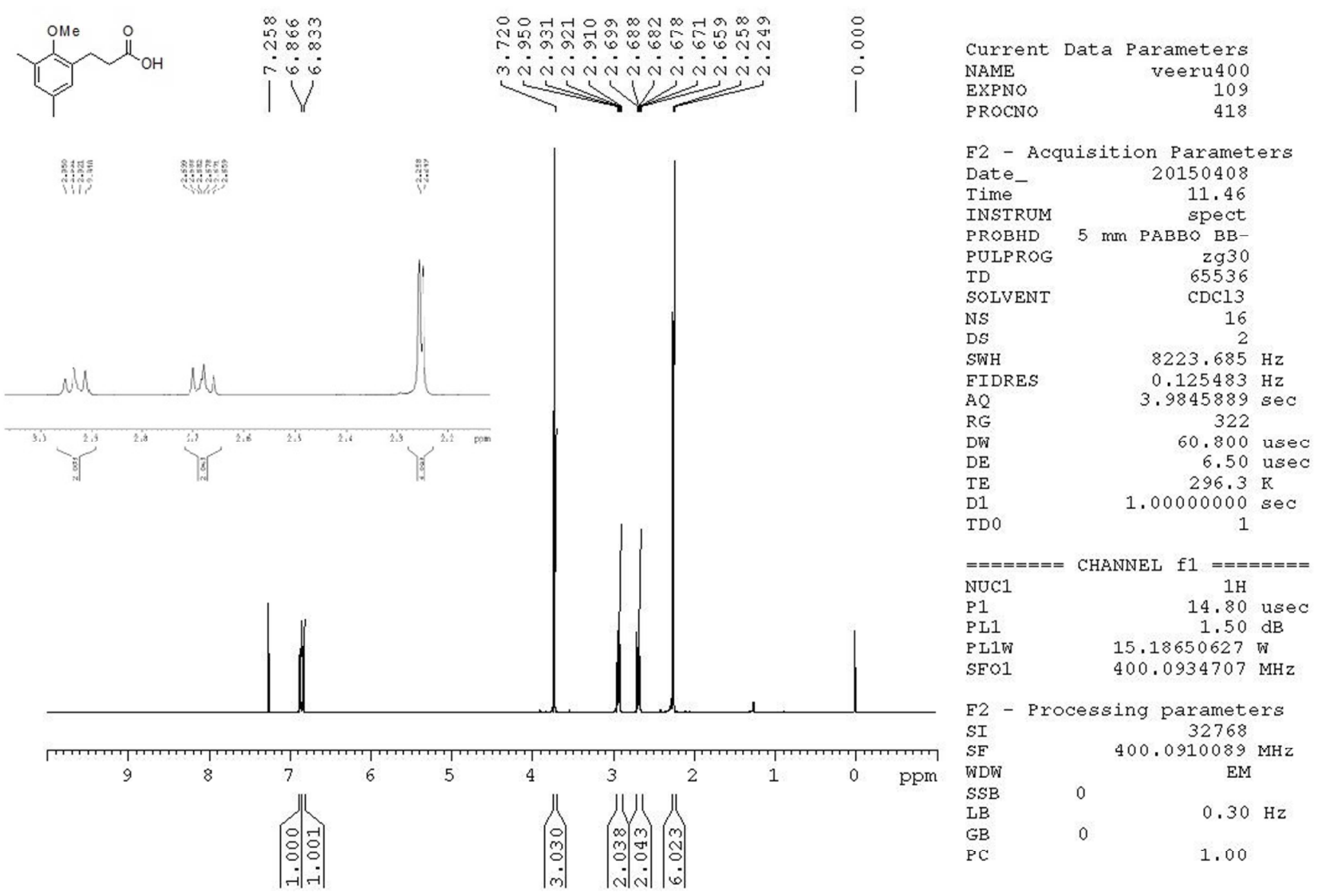

${ }^{1} \mathrm{H}$ NMR (400MHz, $\mathrm{CDCl}_{3}$ ) Spectrum of compound (6) 


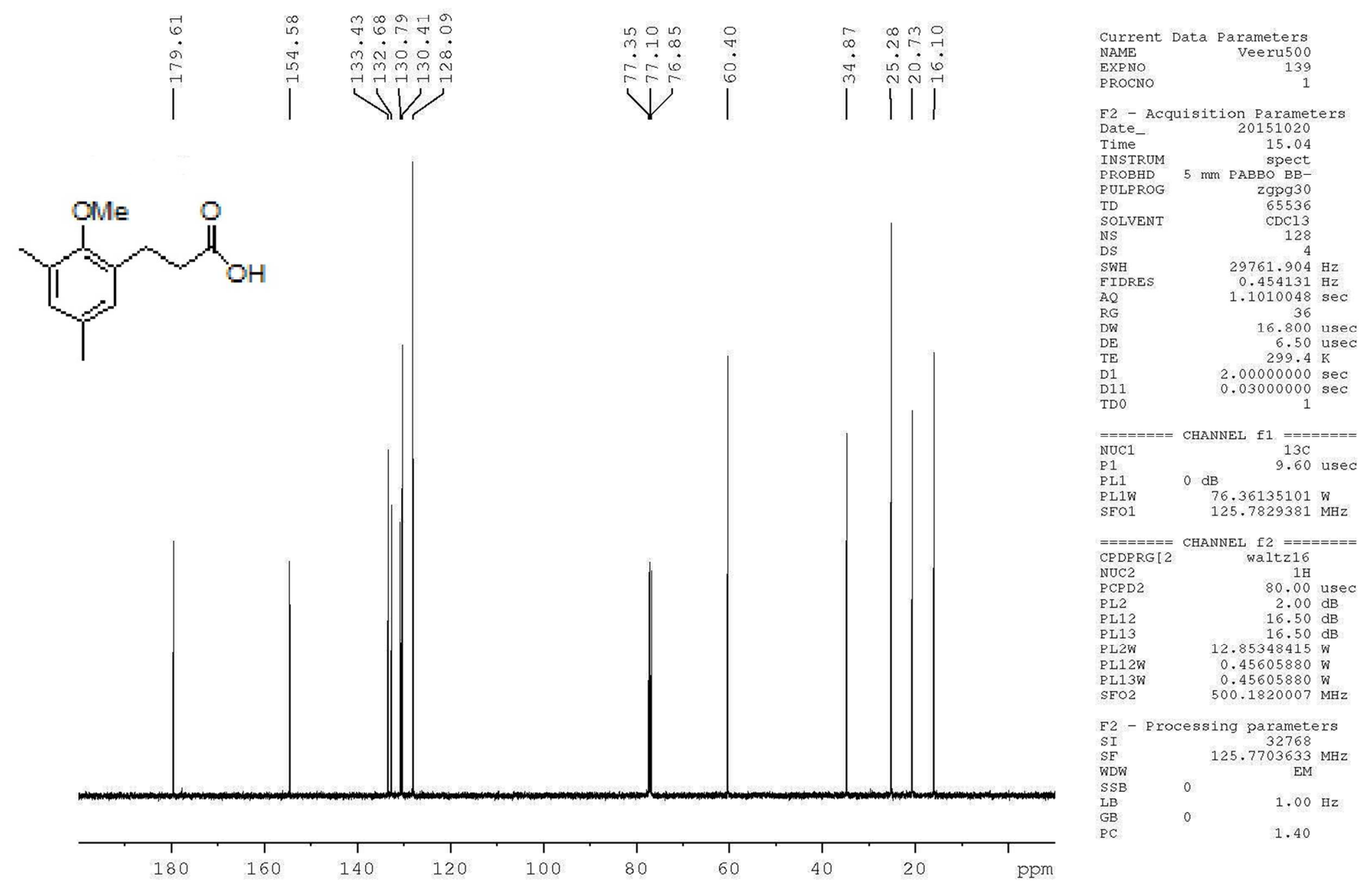

${ }^{13} \mathrm{C}$ NMR (125MHz, $\mathrm{CDCl}_{3}$ ) Spectrum of compound (6) 


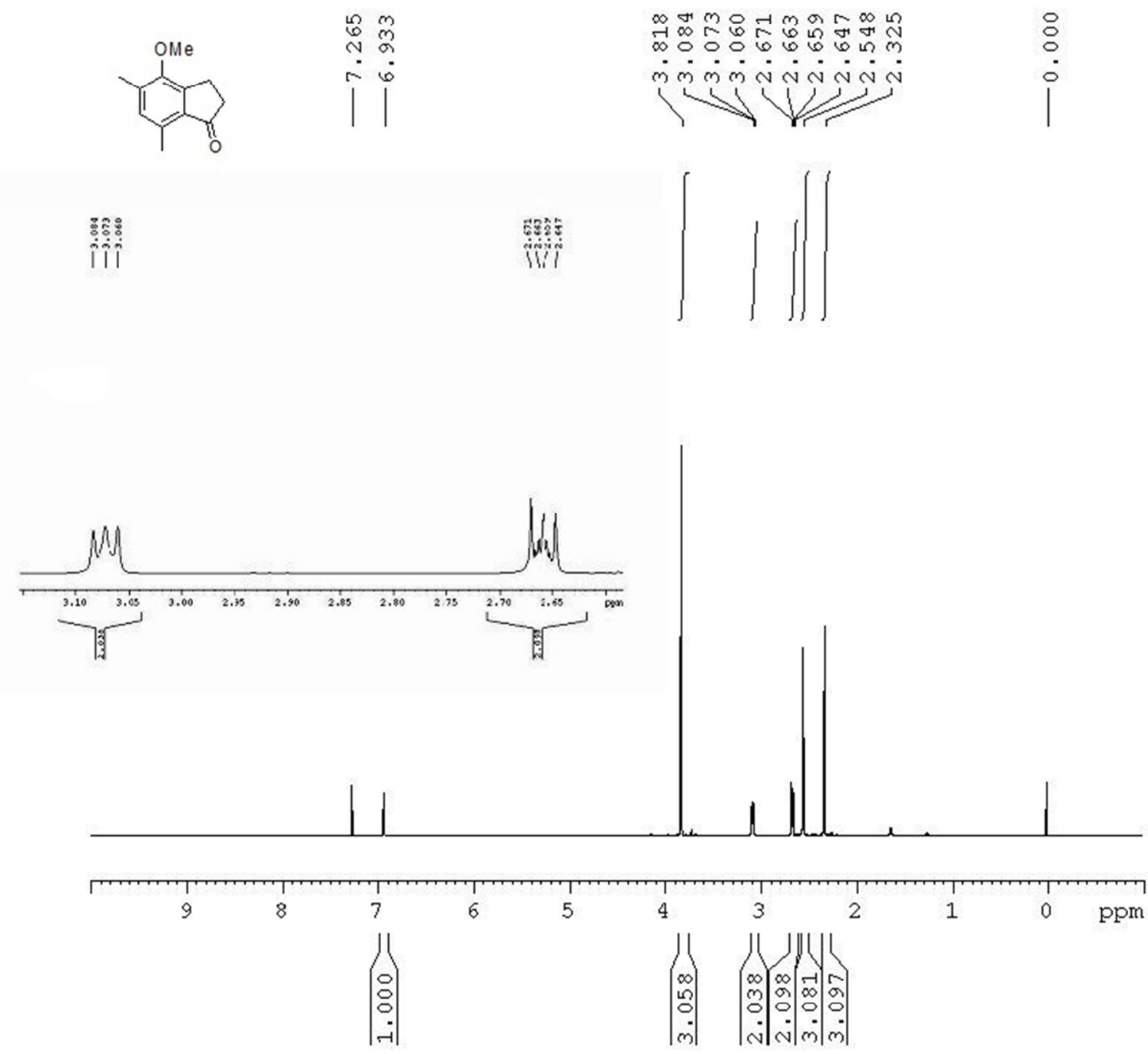

Current Data Parameters

NAME veeru500

EXPNO

PROCNO

86
1

F2 - Acquisition Parameters

Date 20150410

$\begin{array}{ll}\text { Time } & 10.24\end{array}$

INSTRUM spect

PROBHD $5 \mathrm{~mm}$ IXI $1 \mathrm{H} / \mathrm{D}-$

PULPROG

$T D$

SOLVENT

NS

DS

SWH

FIDRES

AQ

RG

DW

$\mathrm{DE}$

TE $z g 30$
65536

$\mathrm{DCl} 3$

16

$10330.578 \mathrm{~Hz}$

$0.157632 \mathrm{~Hz}$

$3.1719425 \mathrm{sec}$ 228

48.400 usec 6.50 usec $295.9 \mathrm{~K}$

$1.00000000 \mathrm{sec}$ TDO 1

$========$ CHANNEL fI

NUC1

$===$

P1

PL1

PLIW

7.00 usec 7.00

$15.45328617 \mathrm{~W}$

F2 - Processing parameters

SI 32768

SF $\quad 500.1800085 \mathrm{MHz}$

WDW $\mathrm{EM}$

SSB $\quad 0$

$30 \mathrm{~Hz}$

GB

1.00

${ }^{1} \mathrm{H}$ NMR (500MHz, $\mathrm{CDCl}_{3}$ ) Spectrum of compound (3) 
<smiles>COc1c(C)cc(C)c2c1CCC2=O</smiles>

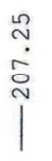
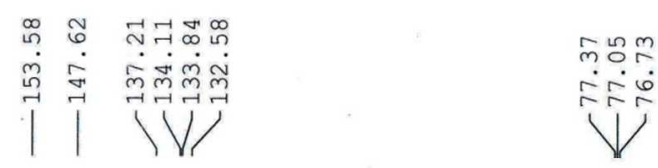

ตั่

$\left.\right|^{\infty}$

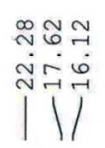

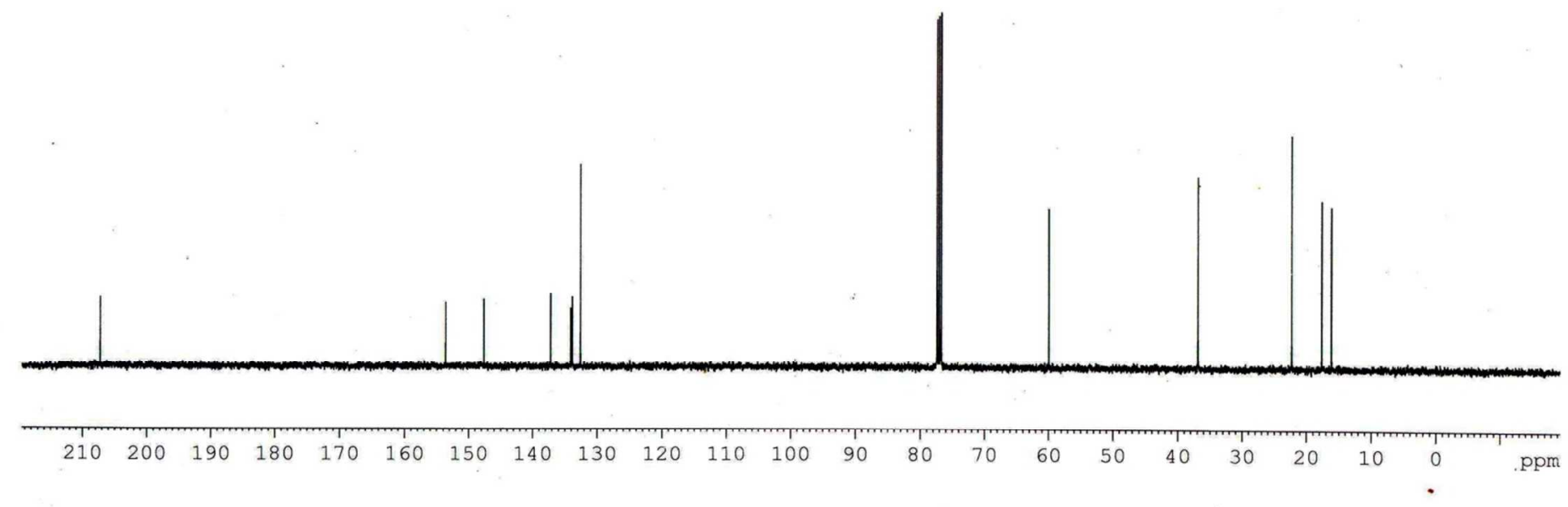

${ }^{13} \mathrm{C}$ NMR (100MHz, $\mathrm{CDCl}_{3}$ ) Spectrum of compound (3) 


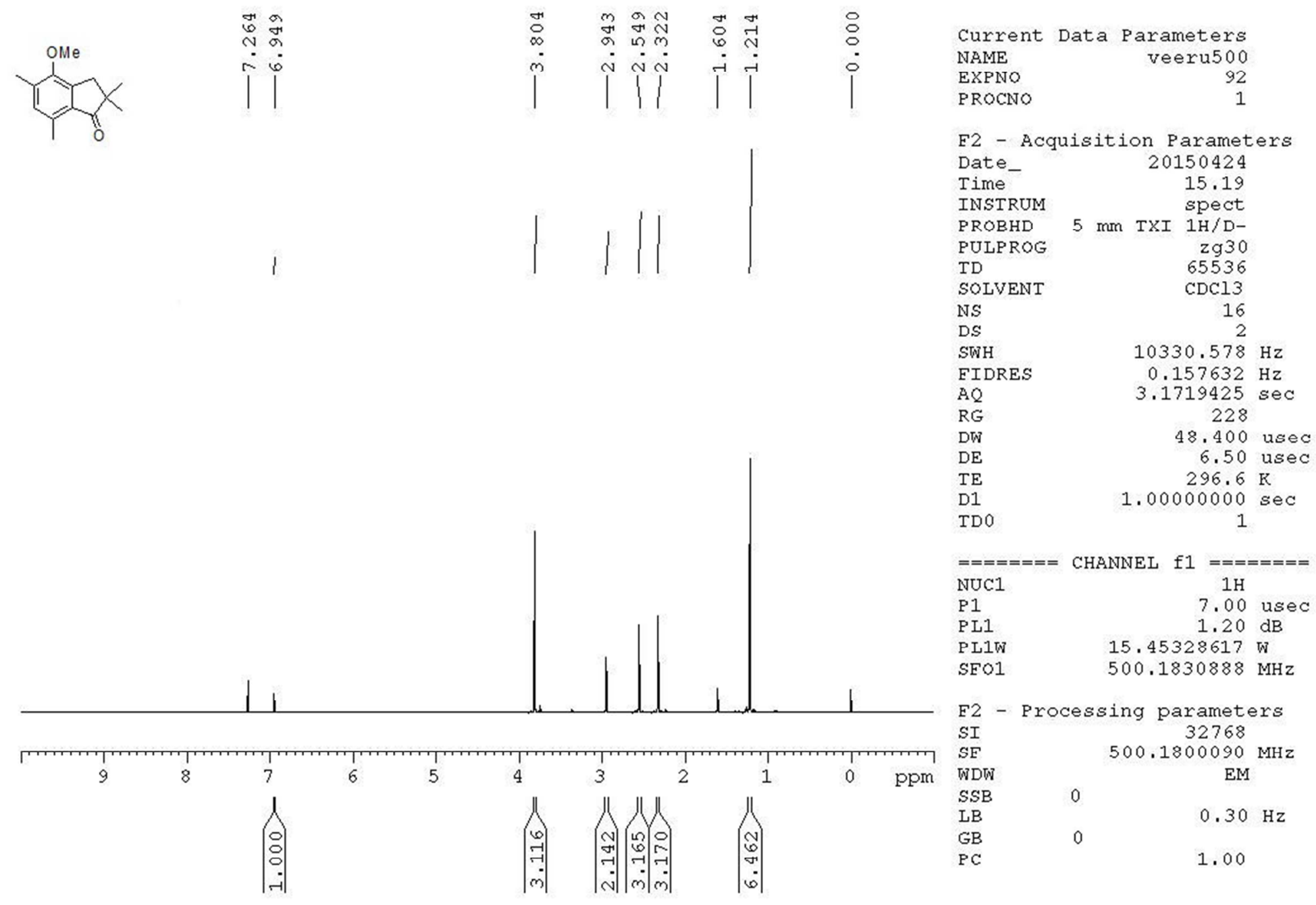

${ }^{1} \mathrm{H}$ NMR (500MHz, $\mathrm{CDCl}_{3}$ ) Spectrum of compound (7) 


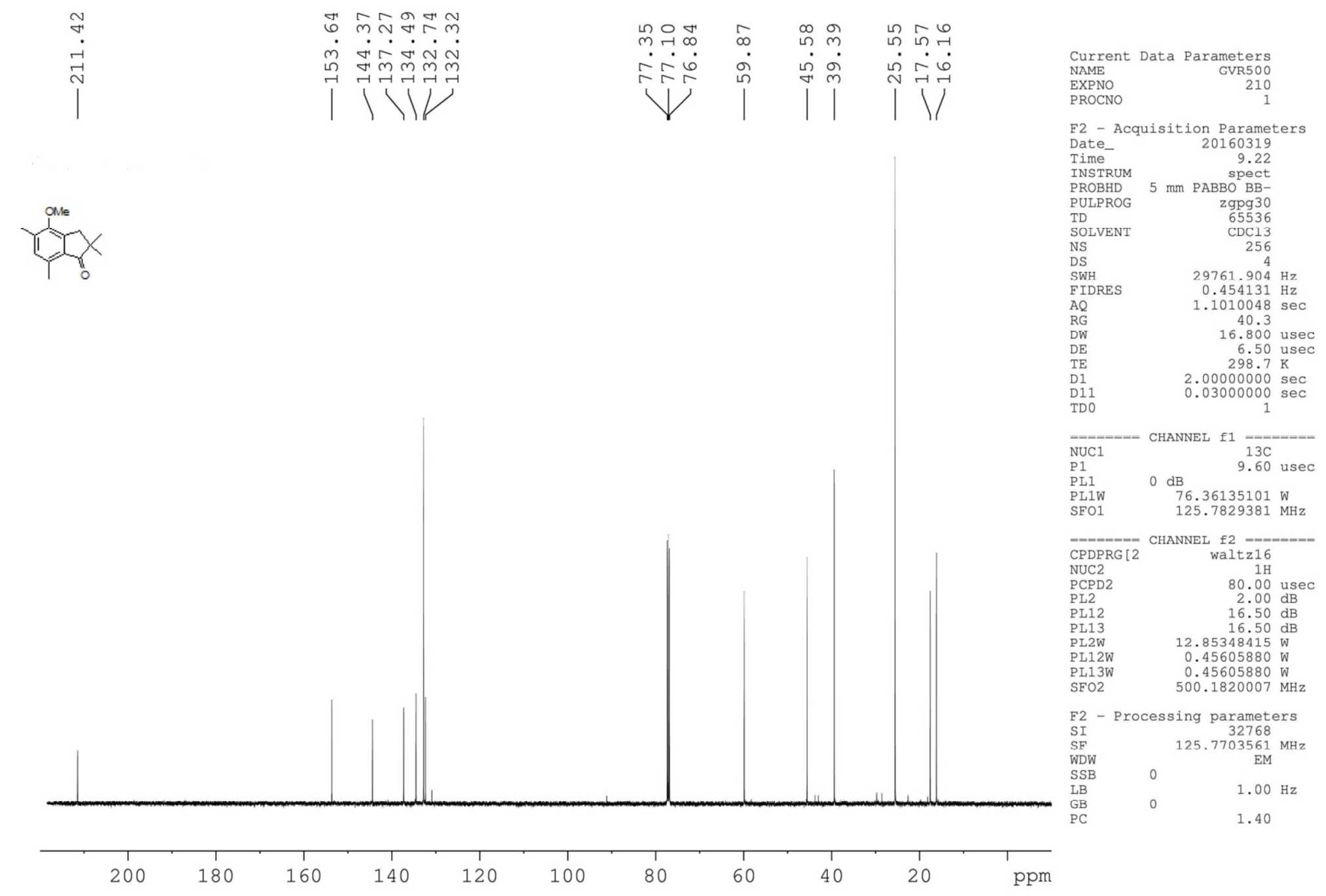

${ }^{13} \mathrm{C}$ NMR (125MHz, $\mathrm{CDCl}_{3}$ ) Spectrum of compound (7) 

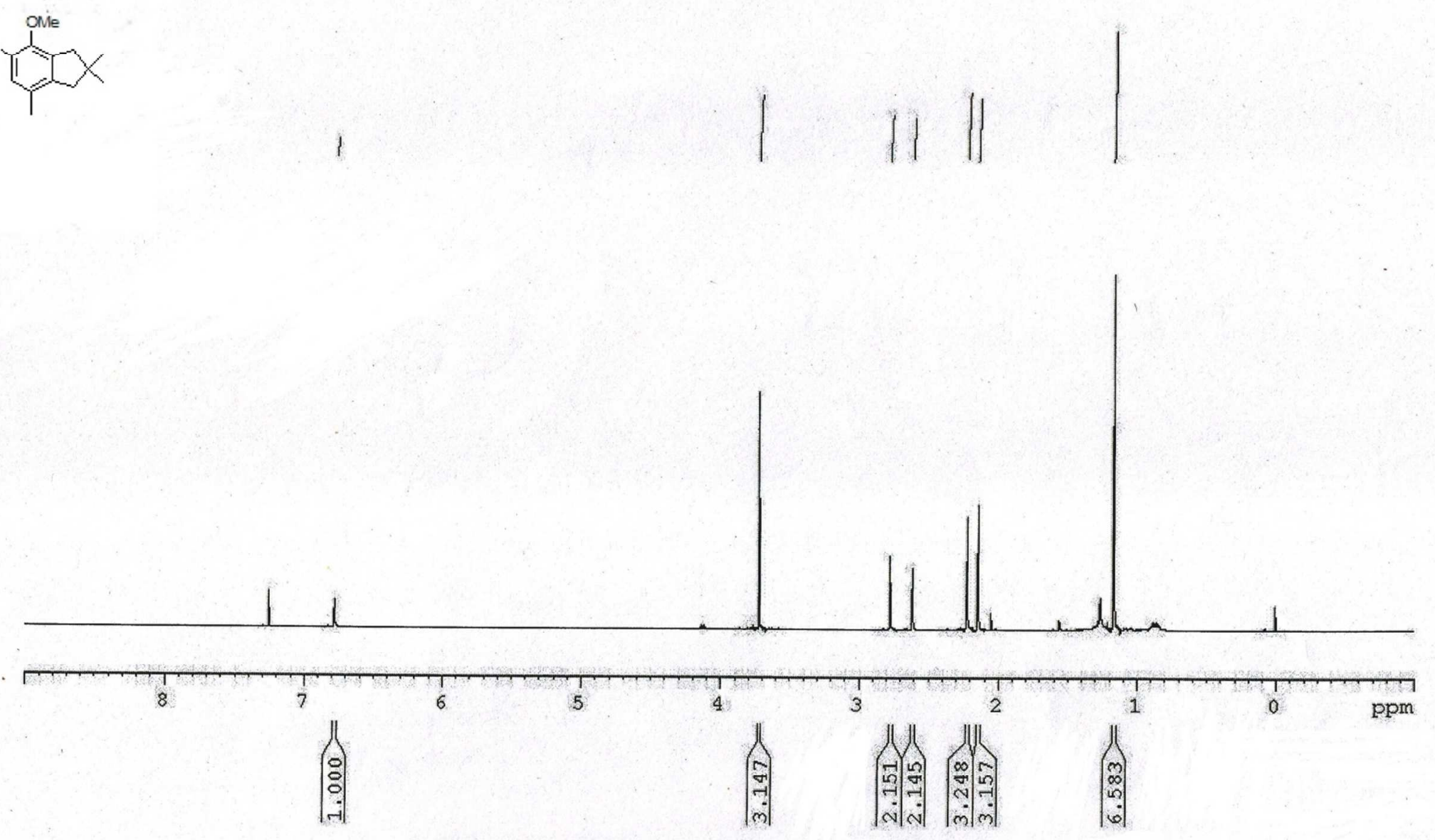

${ }^{1} \mathrm{H}$ NMR (500MHz, $\mathrm{CDCl}_{3}$ )Spectrum of compound (2) 


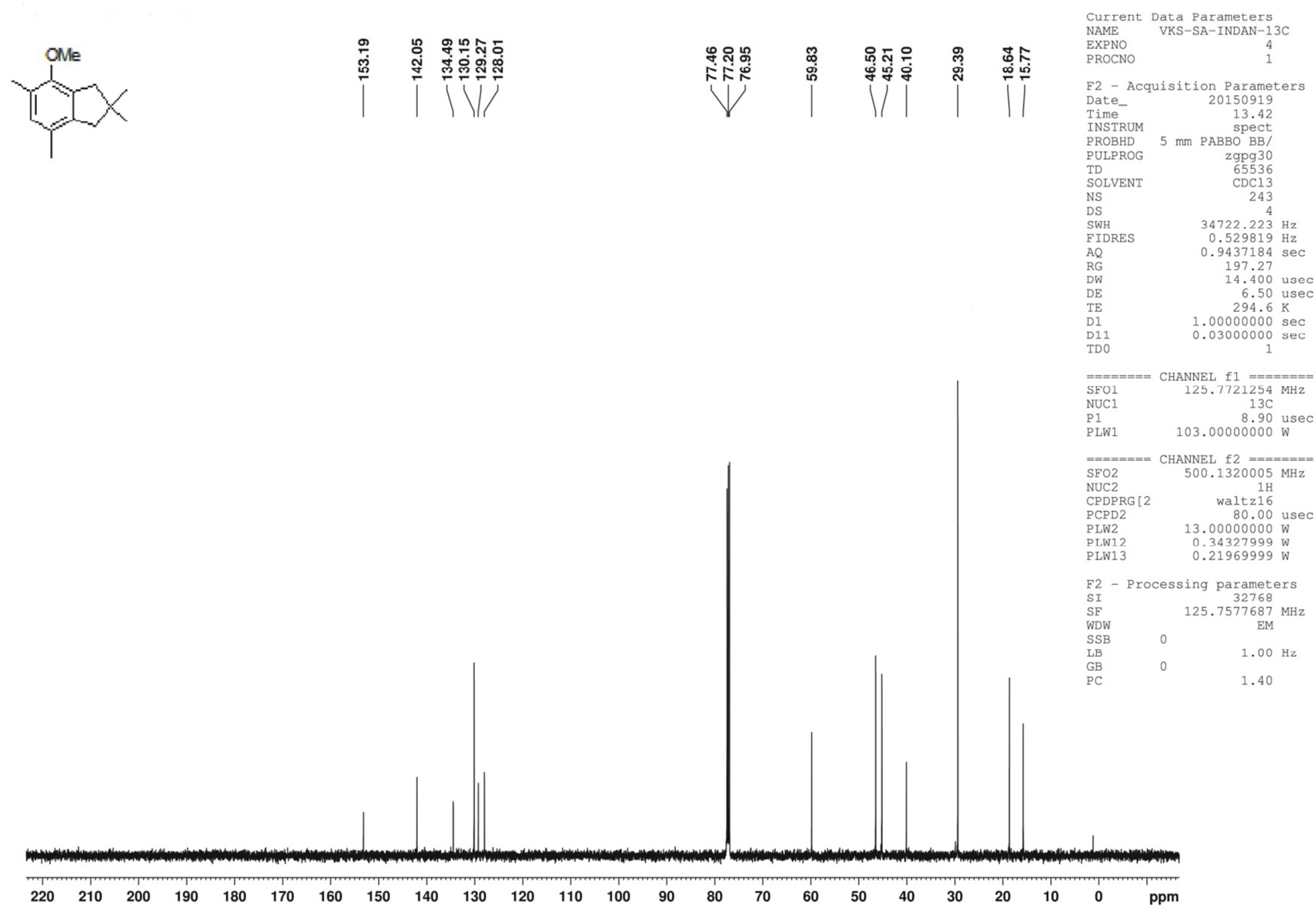

${ }^{13} \mathrm{C}$ NMR (125 MHz, $\mathrm{CDCl}_{3}$ ) Spectrum of compound (2) 


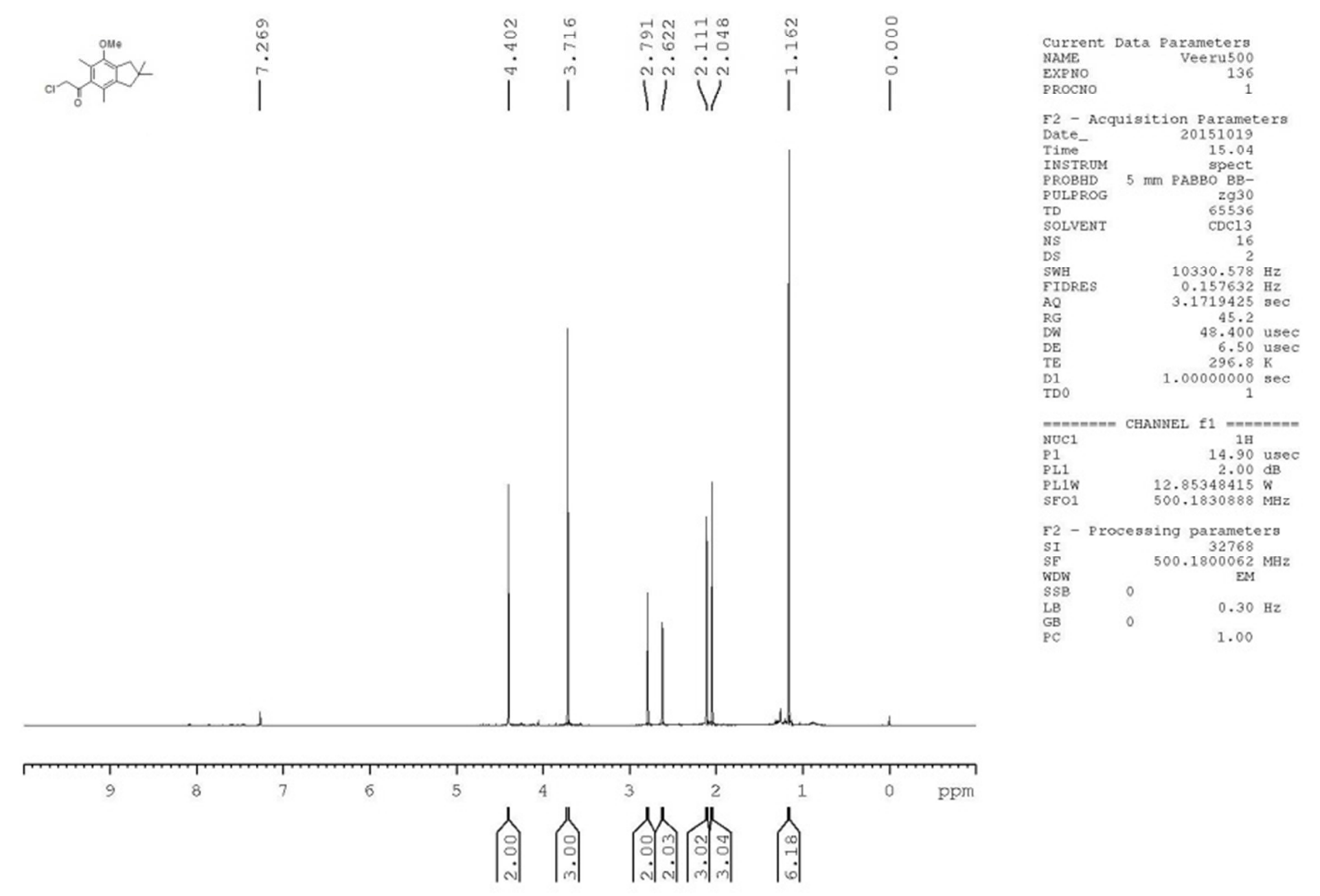

${ }^{1} \mathrm{H}$ NMR (500MHz, $\mathrm{CDCl}_{3}$ ) Spectrum of compound (8) 


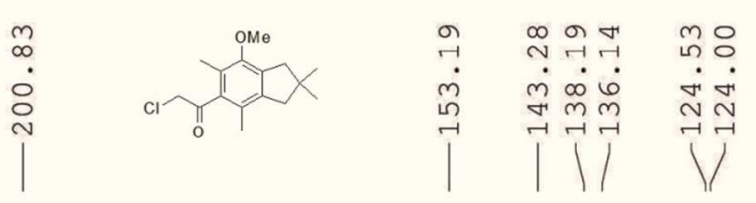

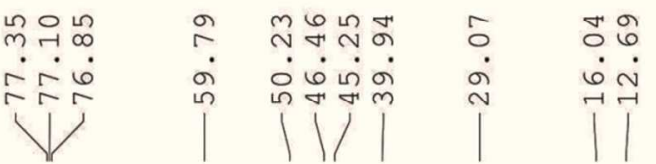

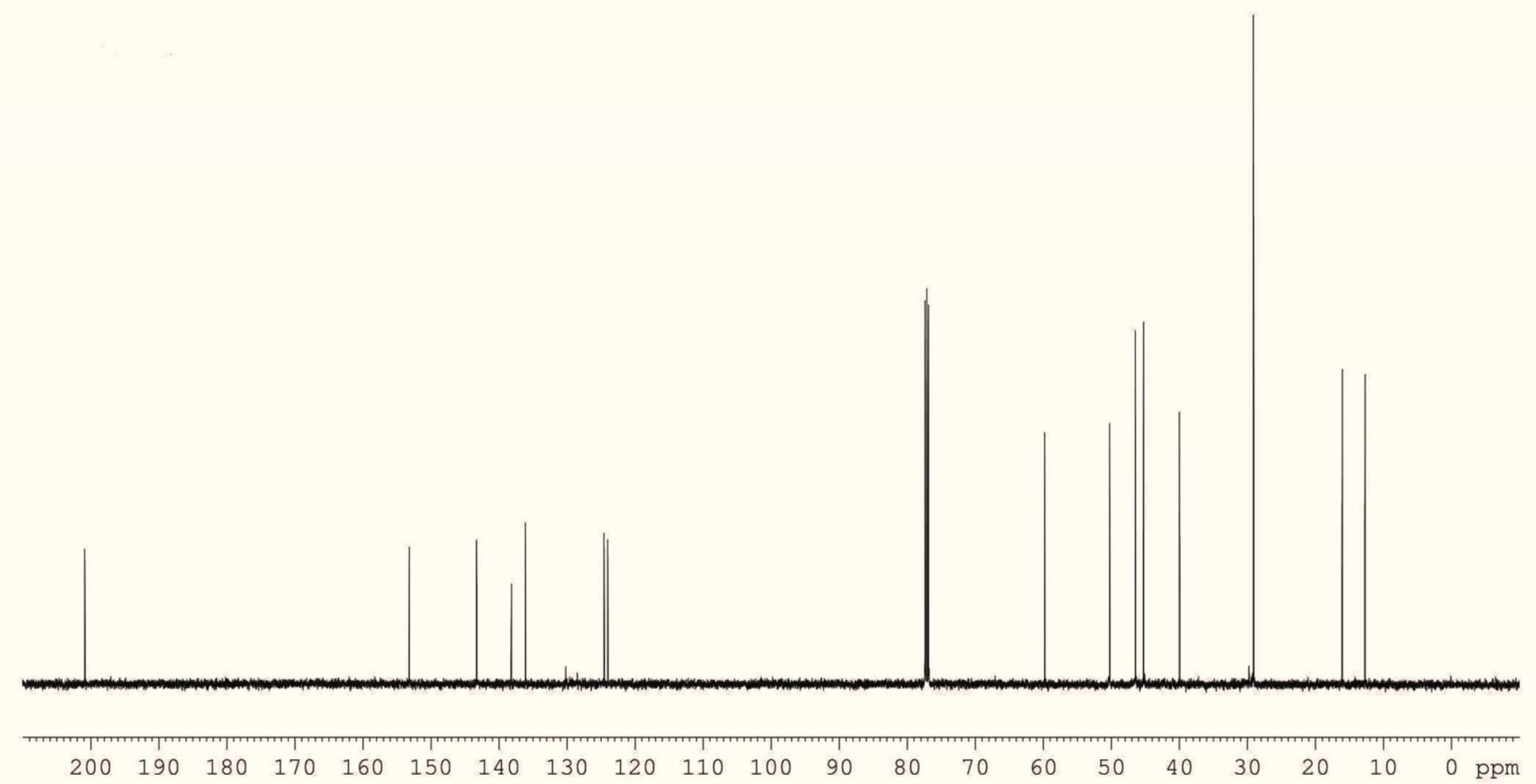

${ }^{13} \mathrm{C}$ NMR (125 MHz. $\mathrm{CDCl}_{2}$ ) Spectrum of compound (8) 


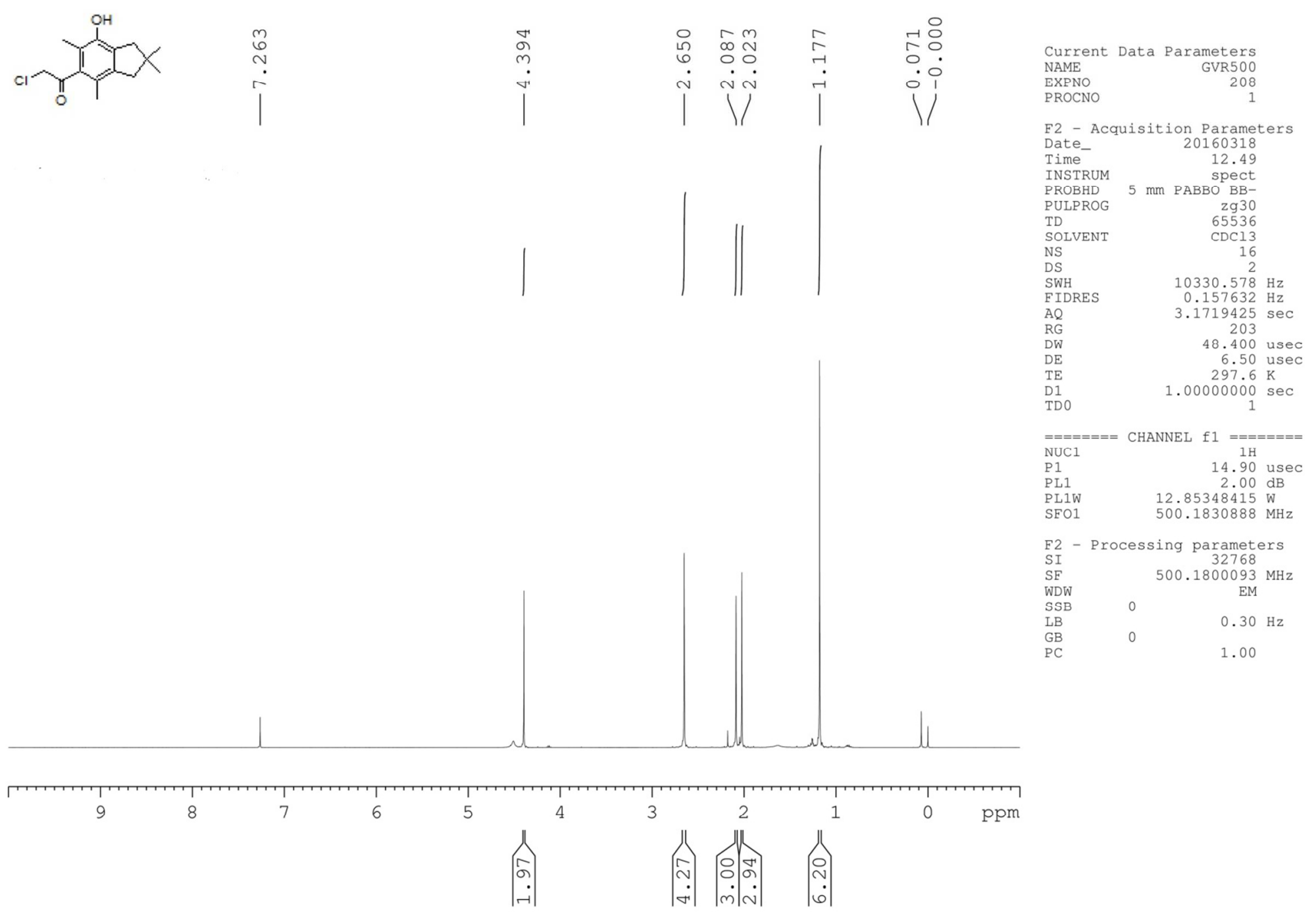

${ }^{1} \mathrm{H}$ NMR (500MHz, $\mathrm{CDCl}_{3}$ ) Spectrum of compound (11) 


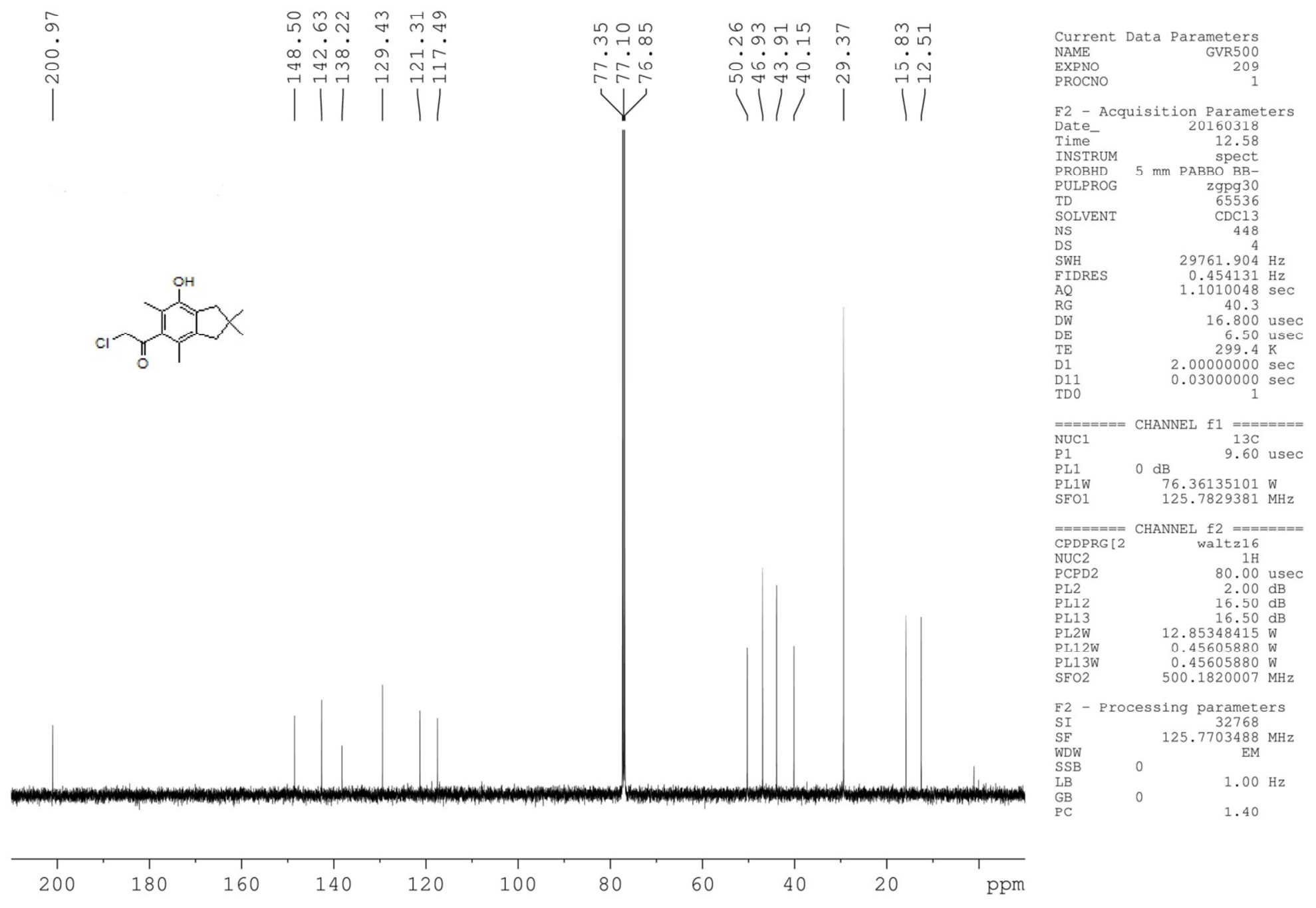

${ }^{13} \mathrm{C}$ NMR (125 MHz, $\mathrm{CDCl}_{3}$ ) Spectrum of compound (11) 


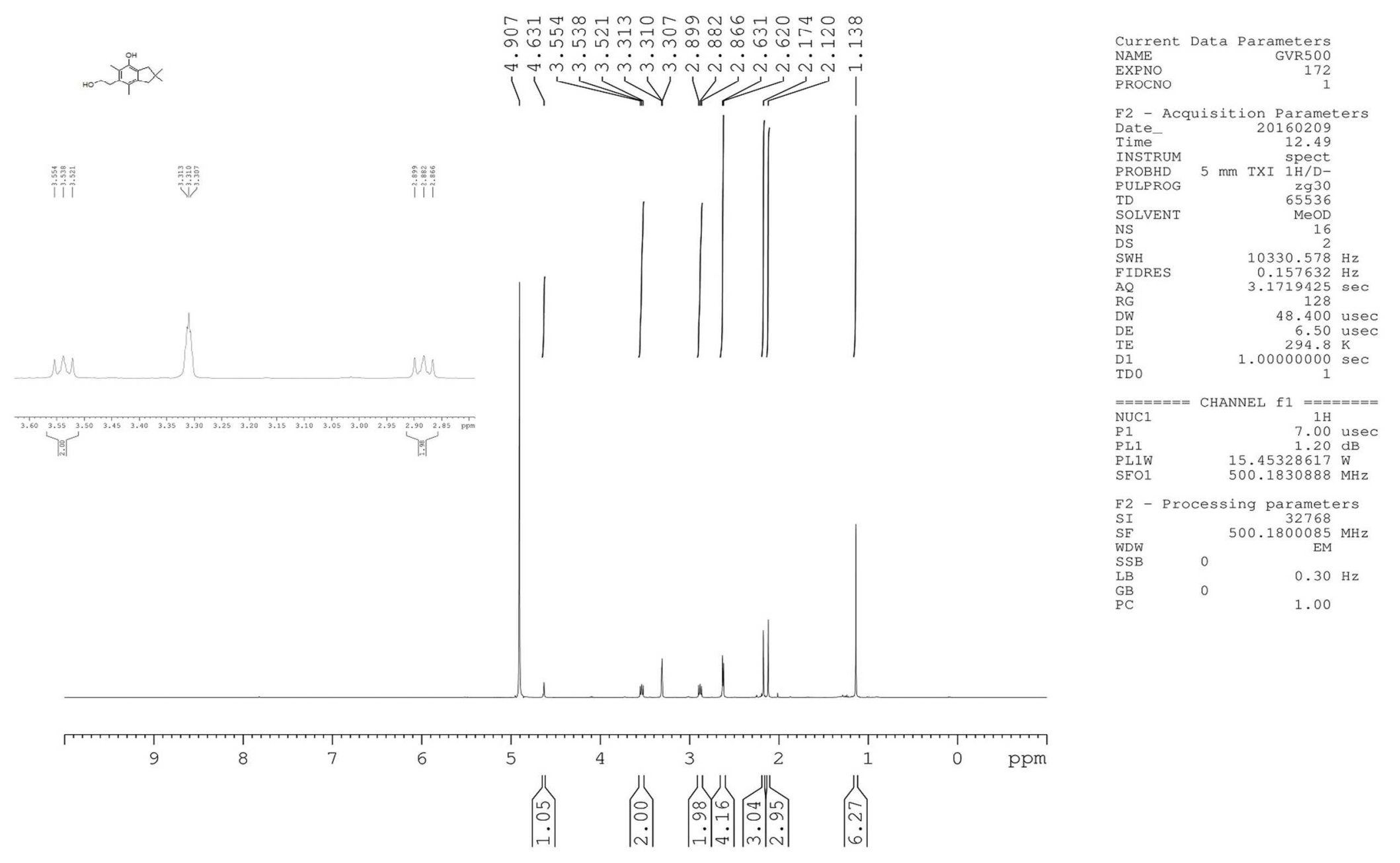

${ }^{1} \mathrm{H}$ NMR (500MHz, $\mathrm{CD}_{3} \mathrm{OD}$ ) Spectrum of compound (1) 

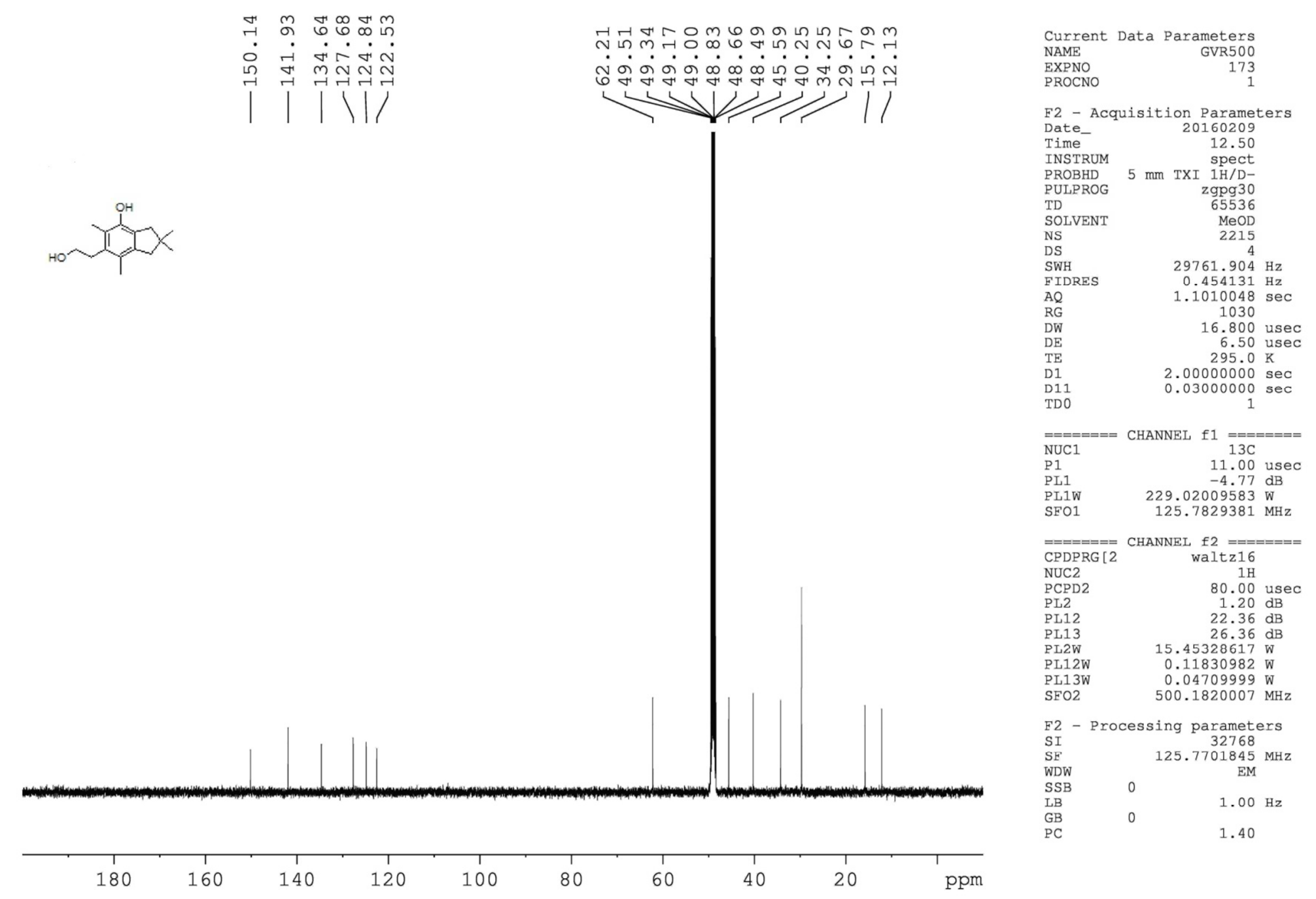

${ }^{13} \mathrm{C}$ NMR (125 MHz, CD $\left.{ }_{3} \mathrm{OD}\right)$ Spectrum of compound (1) 


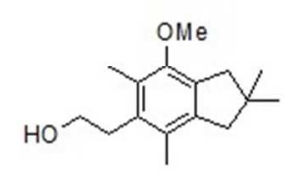

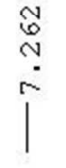
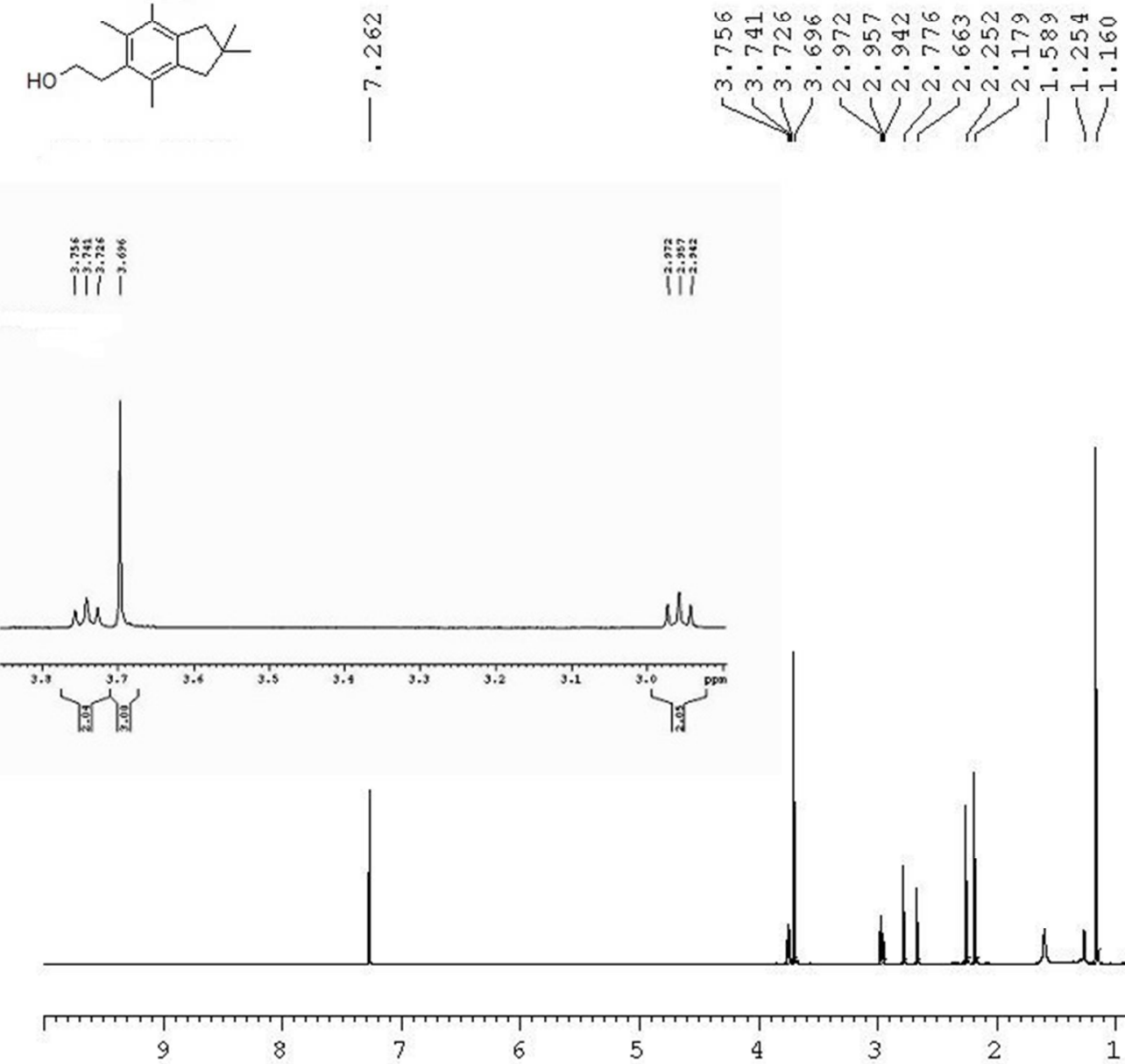
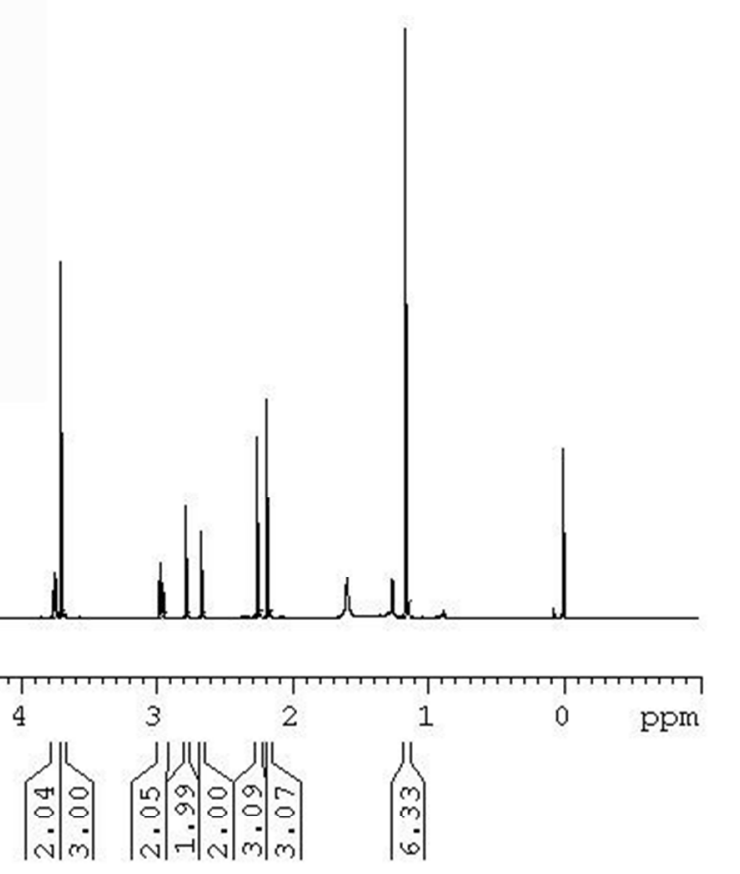

Current Dats Farameter

Veerv-500

PROCNO

130

F2 - Acquizition Parameter

Dste-

20150914
11.45

INSTRUM

PROBHD 5 Ju FABBO BP

PULPROG $\quad$ zg30

TD

SOL

NS
DS
SWH
FIDRE

FIDRES $\quad 10330.578 \mathrm{~Hz}$

AQ $\quad 0.157632 \mathrm{~Hz}$

RG $\quad 322 \mathrm{sec}$

$\begin{array}{lr}\mathrm{D} & 48.400 \\ \mathrm{DE} & 5.50 \mathrm{uec}\end{array}$

TE $295.9 \mathrm{~K}$

D1 $\quad 1.00000000 \mathrm{sec}$

$=======0$ CHANNEL f1 $=======$

$\begin{array}{lrl}\text { NuC1 } & 1 \mathrm{H} \\ \mathrm{P1} & 14.90 \text { usec } \\ \text { PL1 } & 2.00 \mathrm{~dB}\end{array}$

$\begin{array}{lr}\text { PL1 } & 2.00 \mathrm{~dB} \\ \text { PL1W } & 12.85348415 \mathrm{w}\end{array}$

$500.1830888 \mathrm{MH}$

F2 - Processing parameters

$\begin{array}{ll}\text { SI } & 32768 \\ \text { SF } & 500.1800101 \\ \text { NDW } & \end{array}$

SSE 0

$\begin{array}{lll}\mathrm{LB} & 0.30 \\ \mathrm{GC} & & 1.00\end{array}$

${ }^{1} \mathrm{H}$ NMR (500MHz, $\mathrm{CDCl}_{3}$ ) Spectrum of compound (9) 


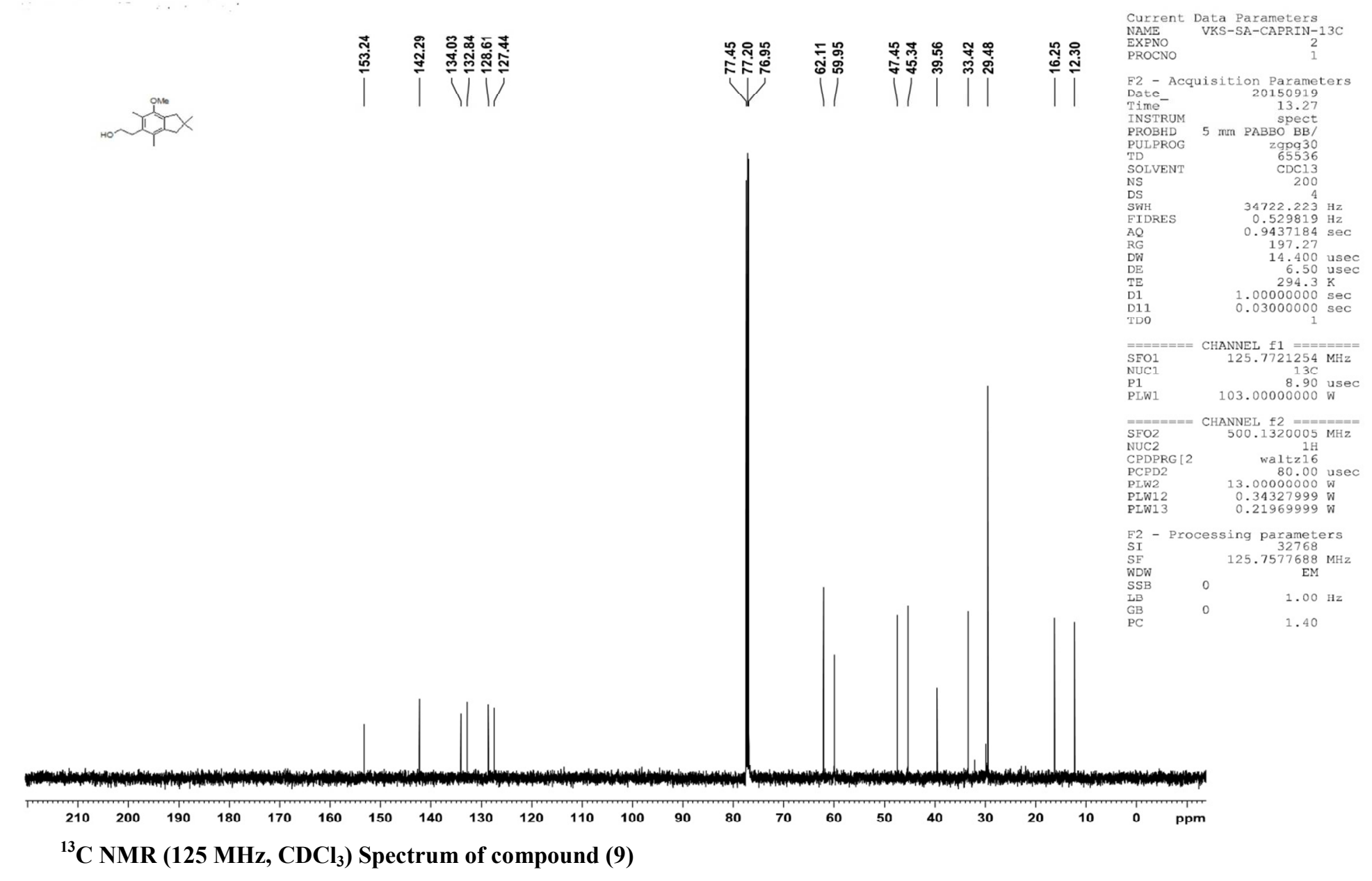




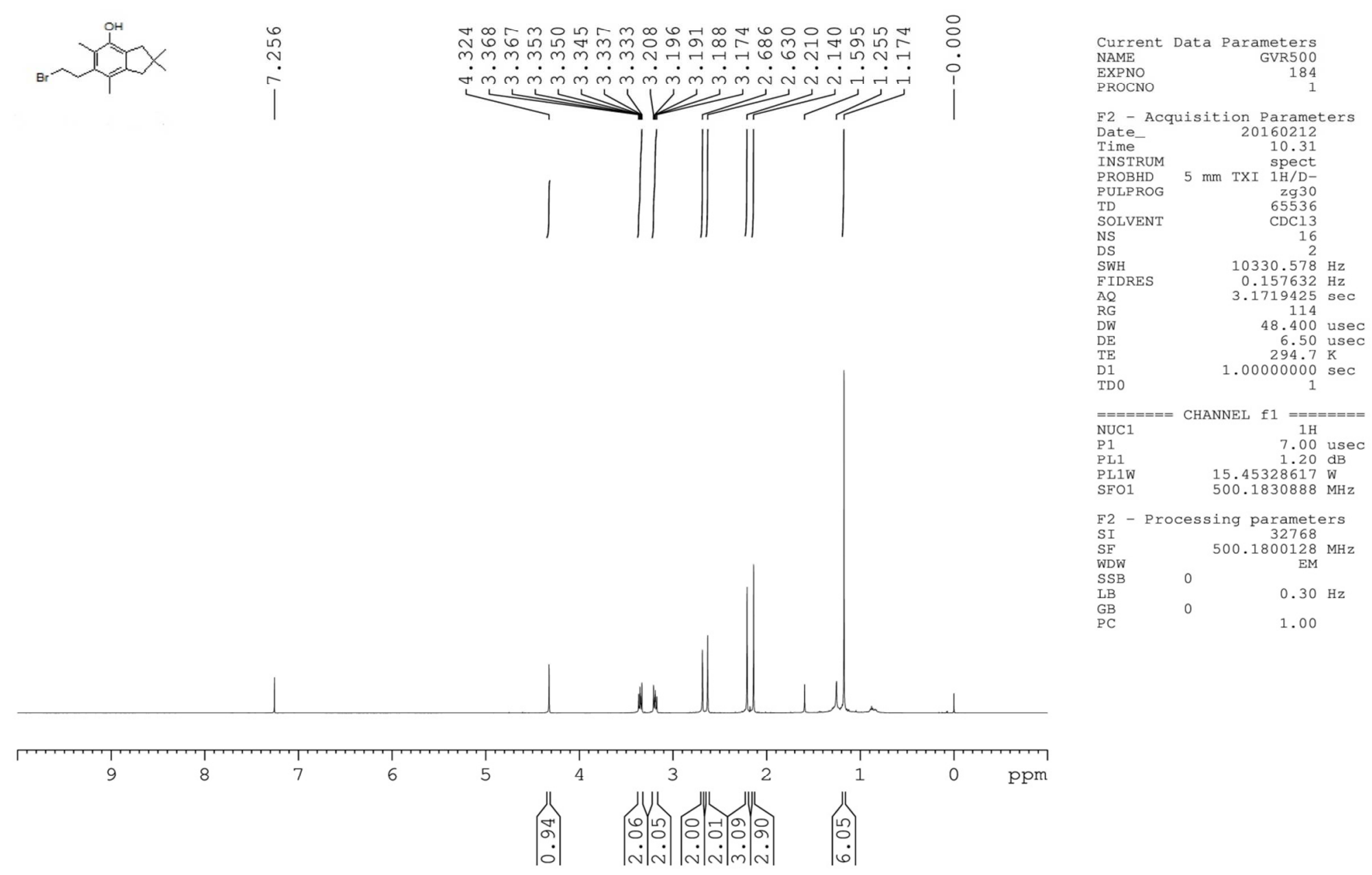

${ }^{1} \mathrm{H}$ NMR (500MHz, $\mathrm{CDCl}_{3}$ ) Spectrum of compound (10) 


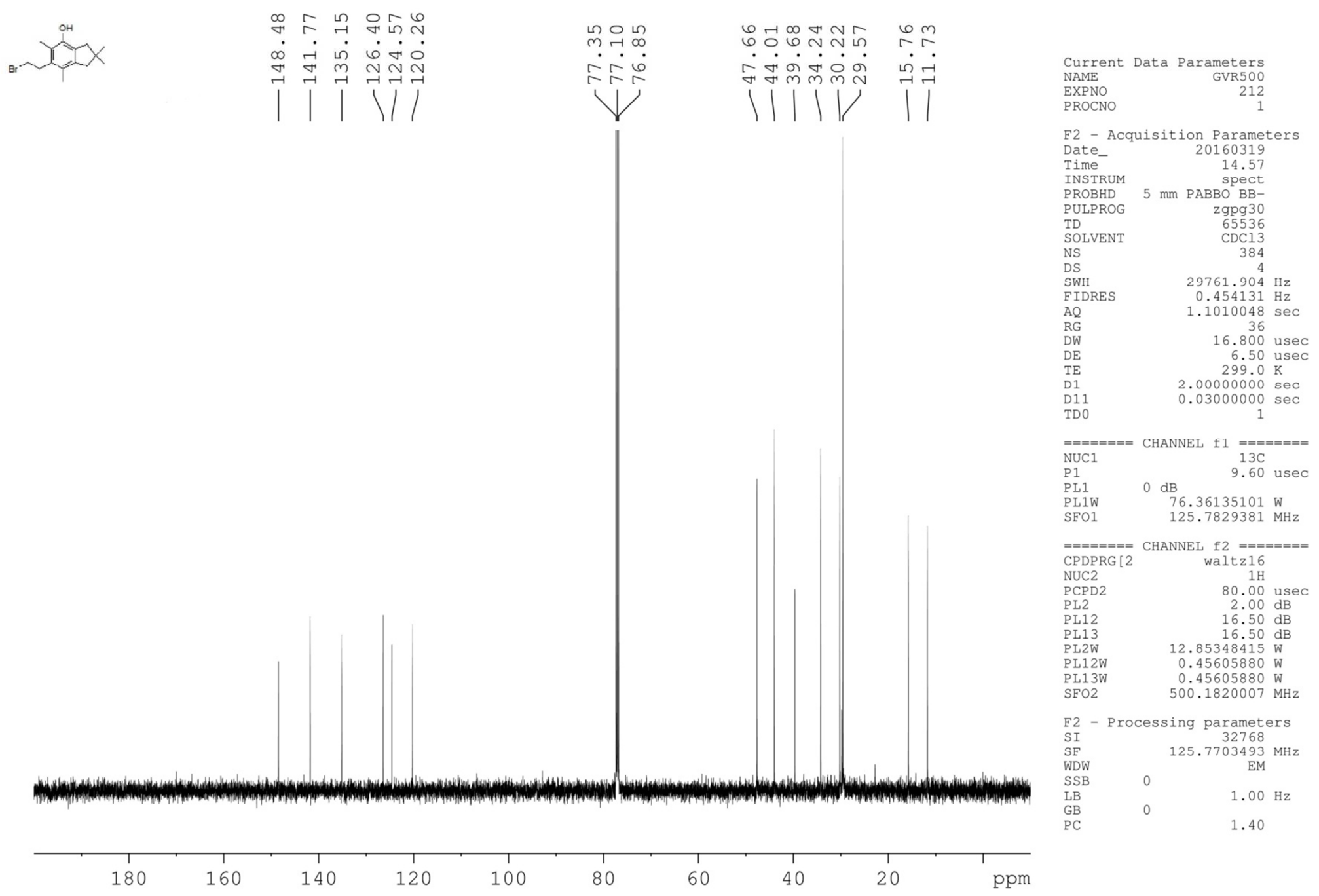

${ }^{13} \mathrm{C}$ NMR (125 MHz, $\mathrm{CDCl}_{3}$ ) Spectrum of compound (10) 